\title{
Growth, Polymorphism, and Spatially Controlled Surface Immobilization of Biotinylated Variants of IAPP ${ }_{21-27}$ Fibrils
}

Torsten John, Juhaina Bandak, Nilushiya Sarveson, Claudia Hackl, Herre Jelger Risselada, Andrea Prager, Christian Elsner, and Bernd Abel*

Cite This: Biomacromolecules 2020, 21, 783-792

Read Online

ABSTRACT: The islet amyloid polypeptide (IAPP) is a regulatory peptide that can aggregate into fibrillar structures associated with type 2 diabetes. In this study, the IAPP ${ }_{21-27}$ segment was modified with a biotin linker at the N-terminus (BtnGNNFGAIL) to immobilize peptide fibrils on streptavidin-coated surfaces. Key residues for fibril formation of the $\mathrm{N}$-terminal biotinylated $\mathrm{IAPP}_{21-27}$ segment were identified by using an alanine scanning approach combined with molecular dynamics simulations, thioflavin $\mathrm{T}$ fluorescence measurements, and scanning electron microscopy. Significant contributions of phenylalanine (F23), leucine (L27), and isoleucine (I26) for the fibrillation of the short peptide segment were

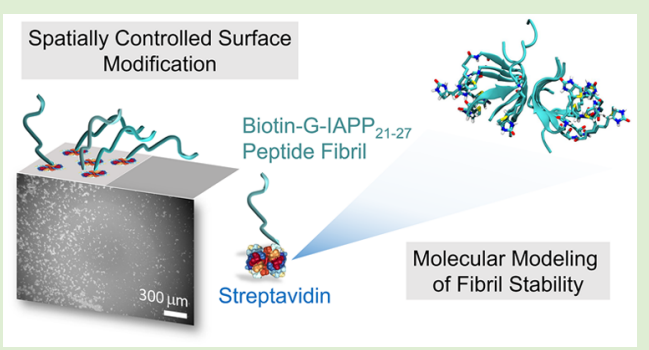
identified. The fibril morphologies of the peptide variants differed depending on their primary sequence, ranging from flexible and semiflexible to stiff and crystal-like structures. These insights could advance the design of new functional hybrid bionanomaterials and fibril-engineered surface coatings using short peptide segments. To validate this concept, the biotinylated fibrils were immobilized on streptavidin-coated surfaces under spatial control.

\section{INTRODUCTION}

The assembly of soluble peptide or protein monomers into fibrillar supramolecular structures, consisting of intermolecular hydrogen-bonded $\beta$-sheets, is associated with serious degenerative diseases, such as Alzheimer's disease, type 2 diabetes, and other misfolding disorders. ${ }^{1,2}$ Despite their physiological role, amyloid fibrils are promising tools for applications in nanoelectronics, biosensing, and separation technologies. ${ }^{3-7}$ The preparation of amyloid fibrils in bulk quantities, from readily available low-cost materials, could increase their commercial potential in the near future. ${ }^{8}$ The advantage to control the properties of these supramolecular structures by tailoring the primary peptide sequence of the monomers is a unique feature which enables a high flexibility in material design.

The ability to control fibril morphology and fibrillation kinetics enables to tailor their specific properties and function. 6,9 Amyloid fibril morphologies can differ in length, diameter, and twist angle. ${ }^{10-1 \frac{1}{2}}$ It has been shown that the length of fibrils influences its cytotoxicity and has an impact on the membrane stability in a physiological environment. ${ }^{13,14}$ The fibril morphology is determined by its flexibility or stiffness that results from the intermolecular forces and amino acid side-chain properties. ${ }^{15,16} \mathrm{~A}$ uniform or homogeneous morphology of fibrils is hardly achievable in vitro. ${ }^{17}$ Amyloid fibrils exist in a variety of structural forms, and it has been shown that the alteration of the solution conditions may favor a particular morphology. 8,18 For technical applications, the fibrillation time and the option to control the fibrillation process, e.g., to trigger the start of fibrillation, play a crucial role. Light- or $\mathrm{pH}$-stimulated fibrillation processes have been investigated to address this topic. ${ }^{19,20}$

In this study, we investigated the influence of amino acid substitutions in the primary peptide sequence of N-terminalmodified NNFGAIL peptides $\left(\mathrm{IAPP}_{21-27}\right)$ on the kinetics and morphology of fibrillar structures. IAPP (islet amyloid polypeptide, amylin) is associated with type 2 diabetes (Figure 1A.i). ${ }^{21,22}$ The identification of residues, which are less relevant for fibril formation, could provide suitable targets for the incorporation of functionalities into the peptide, for instance, molecular switches, catalytic centers, labels, or functional binding units. The IAPP $_{21-27}$ peptide (NNFGAIL) is an exception among other steric-zipper amyloid structures reported by the Eisenberg group. ${ }^{23}$ Instead of interdigitated side chains, the F23, G24, and A25 residues from neighboring chains form a close main-chain/main-chain interface. The fibril structure consists of a hydrophobic surface domain formed by the side chains of I26 and L27 of one sheet and F23 of the opposite sheet and a hydrophilic surface domain formed by N21 and N22 of each sheet (Figure 1B). ${ }^{23}$ Alanine scanning of each position in the peptide was performed to determine the

Received: October 23, 2019

Revised: December 20, 2019

Published: December 30, 2019 

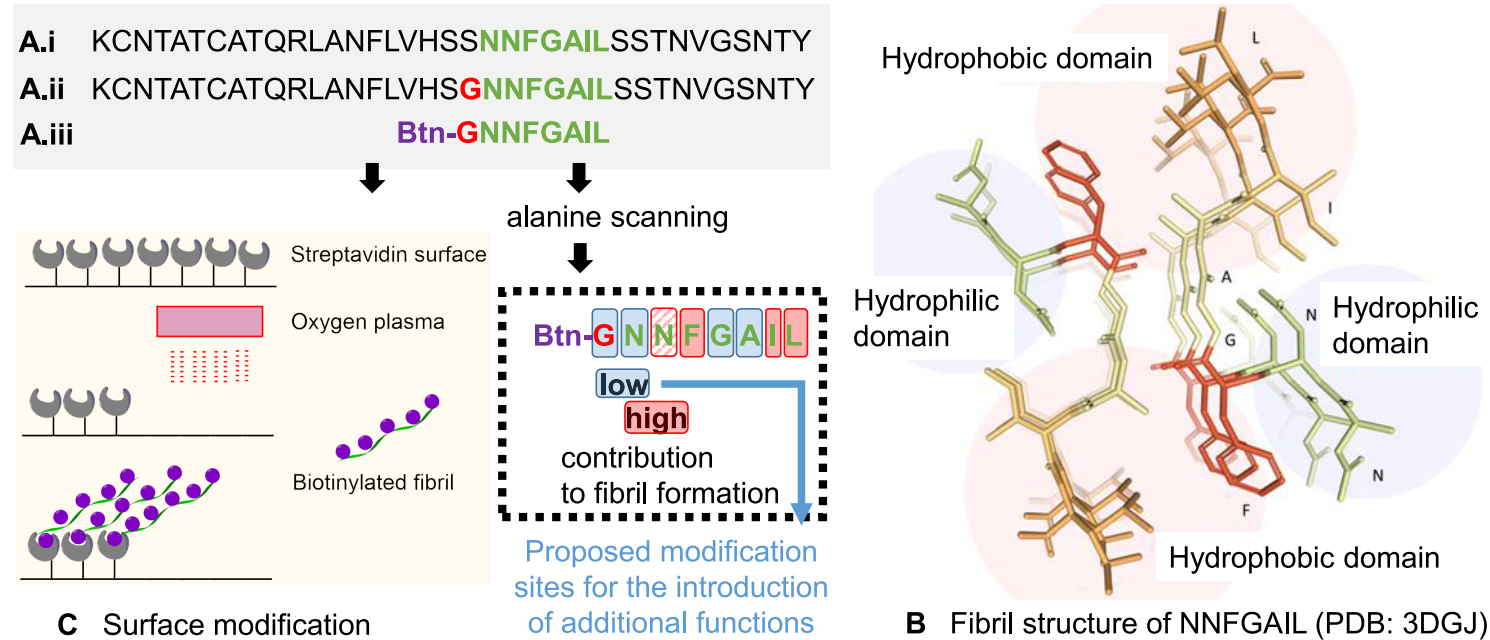

Figure 1. Sequences and structural information about IAPP and IAPP $_{21-27}$ and the relationship to functional properties. (A.i) Sequence of IAPP with the IAPP ${ }_{21-27}$ segment of NNFGAIL as the assumed core structural domain for fibrillation in green. (A.ii) Sequence of an IAPP variation found in an Asian subpopulation with accelerated type 2 diabetes (S20G shown in red). (A.iii) Studied peptide sequence for alanine scanning. (B) Structural representation of a protofibril of the NNFGAIL (PDB: 3DGJ) $)^{23}$ segment from IAPP. Hydrophilic and hydrophobic domains within the protofibril are shown. Molecular data were visualized by using the WebGL-based 3D viewer NGL from the RCSB PDB Web site. ${ }^{29}$ (C) Surface modification strategy (schematic) for the spatial immobilization of biotinylated fibrils.

influence of the corresponding amino acid on the fibrillation process and the morphology of the fibrils. ${ }^{24}$

The peptide variants studied comprise an $\mathrm{N}$-terminal biotinylation of GNNFGAIL (Btn-GNNFGAIL, Figure 1A.iii). The primary function of the $\mathrm{N}$-terminal biotinylation is the generation of an accepted anchor for further modifications by means of the high affinity of the biotinavidin/streptavidin system $\left(K_{\mathrm{d}} \approx 10^{-14} \mathrm{~mol} / \mathrm{L}\right)$ for surface modification processes (Figure 1C). ${ }^{25,26}$ Glycine (G) was introduced at the N-terminal position of NNFGAIL to enlarge the spacer length between the aggregation-prone peptide region and the biotin group for its surface immobilization and to achieve possibly a highly aggregating peptide (Figure 1A.iii). A naturally occurring polymorphic S20G mutation rendered IAPP more aggregation-prone, and an Asian subpopulation with this mutation has an early onset of type 2 diabetes (Figure 1A.ii). ${ }^{27,28}$ We performed thioflavin $\mathrm{T}$ (ThT) fluorescence and scanning electron microscopy (SEM) measurements as well as molecular dynamics (MD) simulations to study the role of peptide modifications for the fibrillation kinetics and morphologies. The hydrophobic peptide residues phenylalanine (F23), isoleucine (I26), and leucine (L27) were identified as highly relevant for peptide aggregation. Finally, Btn-GNNFGAIL fibrils were successfully immobilized on streptavidin-coated surfaces as a proof-of-concept to prepare fibril-engineered surfaces.

\section{EXPERIMENTAL SECTION}

Materials. Chemicals were obtained from commercial sources as follows: Fmoc-amino acids, preloaded Fmoc-amino acid-WANG resins, biotin, thioflavin $\mathrm{T}$ (dye to detect amyloid fibrils), TIPS (Sigma-Aldrich, St. Louis, MO); DMF (peptide synthesis grade), piperidine (peptide synthesis grade), DIEA, diethyl ether (>99.5\%) (Carl-Roth, Karlsruhe, Germany); TFA (Merck, Darmstadt, Germany); TBTU, HOBT (Fluka, Seelze, Germany); 2-propanol (HPLC grade), DMSO (ultrapure) (VWR, Leuven, Netherlands); NNFGAIL (Eurogentec, Seraing, Belgium); HFIP (99.9\%, spectrophotometric grade, Acros Organics, Geel, Belgium); Millipore-grade water (MilliQ Integral 5 laboratory purification system, Merck, Darmstadt, Germany).
Peptide Synthesis and MALDI-TOF-MS. Modified peptides were synthesized by microwave-assisted solid phase peptide synthesis (SPPS) based on standard Fmoc chemistry (Fmoc deprotection: 20\% piperidine in DMF; coupling: TBTU/HOBT: 3 equiv, Fmoc amino acid: 2.9 equiv, DIEA: 10 equiv in DMF) using an Initiator+ SP Wave semiautomatic peptide synthesizer (Biotage, Uppsala, Sweden). The peptide was cleaved from the resin in TFA/TIPS/water (95/2.5/2.5, $\mathrm{v} / \mathrm{v} / \mathrm{v}$ ) and precipitated after solvent evaporation in ice-cold diethyl ether. The peptide was washed at least three times in diethyl ether by resuspending and centrifugal precipitation. The identity of the peptides was determined on an Autoflex III MALDI-TOF mass spectrometer (Bruker, Bremen, Germany) in positive reflectron mode using a HCCA matrix Prespotted AnchorChip target (PAC II target). Observed mass-to-charge ratios are presented in Table S1. Standard sample preparation was performed using the dry droplet method according to the Bruker guide for MALDI sample preparation. Peptides were dissolved in HFIP. For the calibration on the target, the Bruker peptide calibration standard or poly(ethylene glycol) MW 1000 (PEG-1000) was used.

Thioflavin T (ThT) Fluorescence Assay. ThT fluorescence assays were performed at a peptide concentration of $1 \mathrm{mg} / \mathrm{mL}$. The final concentration of ThT in the assays was $5 \mu \mathrm{M}$. Assays were performed in polypropylene tubes with a total volume of $1 \mathrm{~mL}$. Peptide stock solutions of $100 \mathrm{mg} / \mathrm{mL}$ in DMSO were prepared. The $\mathrm{DMSO} /$ peptide and aqueous ThT solution were rapidly mixed (vortexed) and kept at $25{ }^{\circ} \mathrm{C}$ in a thermomixer (gentle shaking at $200 \mathrm{rpm}$ ). For fluorescence measurements (TECAN microplate reader infinite 200, Männedorf, Switzerland) after certain time intervals, the vials were vortexed, treated with ultrasound for $5 \mathrm{~min}$, and vortexed; subsequently $200 \mu \mathrm{L}$ aliquots were transferred into the well of a microtiter plate and read out $\left(\lambda_{\text {exc }}=410 \mathrm{~nm}, \lambda_{\mathrm{em}}=485 \mathrm{~nm}, n\right.$ $=3)$. Averages and standard errors of the mean were calculated to present the results.

Scanning Electron Microscopy (SEM) Measurements. Peptide solutions were prepared at a concentration of $0.1 \mathrm{mg} / \mathrm{mL}$ (50 $\mathrm{mg} / \mathrm{mL}$ peptide stock in DMSO was used) and incubated for several days. Droplets of the peptide solutions were deposited onto silicon wafers (Plano, Agar Scientific, \#G3390, Germany) after varying times and air-dried. SEM images were recorded by using an Ultra 55 SEM (Carl Zeiss Microscopy, Oberkochen, Germany).

Spatially Controlled Fibril Surface Immobilization. Streptavidin-coated glass slides (PolyAn 3D-Streptavidin glass slides, LOT: 180626, PolyAn, Berlin, Germany) were exposed to an atmospheric 
oxygen plasma through a mask (Atomflow 500 system, Surfx Technologies, U.S.; for details refer to the Supporting Information). This method yielded areas in which the streptavidin was removed by the plasma. The glass slide was incubated with $1 \mathrm{~mL}$ of fibrillation solution as described in the section Thioflavin $\mathrm{T}(\mathrm{ThT})$ Fluorescence Assay $([$ Btn-GNNFGAIL $]=1 \mathrm{mg} / \mathrm{mL})$. The glass slide was covered with a plastic cover plate to prevent evaporation of the solvent. After 2 days, the glass slide was extensively rinsed with ultrapure water, dried, and then coated with a chromium layer to prevent charge effects during SEM analysis.

Molecular Dynamics Simulations (MD). Structural information for the heptapeptide NNFGAIL was obtained from the Protein Data Bank (entry 3DGJ). ${ }^{23}$ Modifications of NNFGAIL, namely NNGGAGL, GNNFGAIL, and Btn-GNNFGAIL, were prepared by using the Maestro suite 2015 (Schrödinger, New York, NY). The biotin structure was obtained from the Automated Topology Builder (ATB) and Repository (Molid 2212)..$^{30-32}$ The peptides were studied with charged termini at $\mathrm{pH}$ 7. Topology information for the peptides without biotin (NNFGAIL, NNGGAGL, and GNNFGAIL) was included in the applied force field. The biotin-modified peptide (BtnGNNFGAIL) was parametrized by using the ATB according to the Gromos philosophy. The topology information for the amino acids was taken from the force field and for biotin from the ATB (Molid 2212). The amide bond between biotin and peptide was obtained from the ATB (Molid 26960) by generating parameters for a biotinylated lysine. ${ }^{30-32}$

Five-stranded protofibrils with two $\beta$-sheets $(5 \times 2)$ were built for all peptides by using the crystal structure information $\left(P 2_{1} 2_{1} 2_{1}\right)$ known for NNFGAIL as the reference starting structure. ${ }^{23}$ For each system, the protofibril was placed in an octahedral periodic box with minimal distances of at least $1.2 \mathrm{~nm}$ between the solute and the box edges. The simulation boxes with the protofibrils were solvated in explicit water by using the simple point charge (SPC) model. ${ }^{33}$ Sodium chloride was added as physiological salt $(0.15 \mathrm{~mol} / \mathrm{L})$.

MD simulations were performed using GROMACS 4.5.7 $7^{34-37}$ and the GROMOS 54A7 united-atom force field ${ }^{38}$ at $300 \mathrm{~K}$. All peptide bonds were constrained to their equilibrium values by using the LINCS algorithm. ${ }^{39,40}$ Water was constrained by using the SETTLE algorithm. ${ }^{41}$ Both Coulomb and van der Waals interactions were described with a cutoff distance of $1.4 \mathrm{~nm}$ (single cutoff). Interactions were updated every fifth step with the charge-group-based pair list. The reaction-field method was applied to correct for long-range electrostatic interactions (dielectric constant of the reaction field, $\varepsilon=78) .{ }^{42}$ Periodic boundary conditions were applied in all three dimensions. A 2 fs time step was used for the integration of the equations of motion. Simulation settings were adapted from the work of Periole et $\mathrm{al}^{43}$ The protofibril systems were initially energy minimized in two steps in a steepest-descent algorithm-first with the peptide atom positions restrained $\left(100 \mathrm{ps}, k=1000 \mathrm{~kJ} \mathrm{~mol}^{-1} \mathrm{~nm}^{-2}\right)$ and second without any positional restraints (100 ps). The simulations were run in an isothermal-isobaric ensemble (NPT). Peptide and water/ions were independently coupled to a Berendsen temperature thermostat with a reference temperature of $300 \mathrm{~K}$ and a relaxation time of $0.1 \mathrm{ps}^{44}$ The pressure was maintained at 1 bar by coupling the system to a Berendsen isotropic pressure barostat with a relaxation time of $1 \mathrm{ps}$ and compressibility of $4.6 \times 10^{-5}$ bar. $^{44}$ After equilibration of the systems with position restrained peptide backbone $\left(k=100 \mathrm{~kJ} \mathrm{~mol}^{-1} \mathrm{~nm}^{-2}\right)$, the simulations were run in three unbiased repetitions, each with random starting velocities fitted to a Boltzmann distribution. The center-of-mass translation was independently removed for protein and water/ions. The $\mathrm{MD}$ simulations were run for $100 \mathrm{~ns}$ to study the protofibril stability.

Simulation trajectories were visualized by using $\mathrm{VMD}^{45}$ and analyzed using GROMACS tools. Either representative or averaged data of the three repetitions were shown and plotted by using OriginPro 8G (OriginLab, Northampton, MA). Clustering analysis of the last $10 \mathrm{~ns}$ of simulation time of all three repetitions each $(3 \times$ $10 \mathrm{~ns}$ ) was performed for all variants, and the central structure of the largest cluster was visualized in VMD as the most dominant structure (linkage method, RMSD cutoff $0.2 \mathrm{~nm}$ ). Root-mean-square deviations
(RMSD) of the peptide backbone were calculated in comparison to the starting structure. RMSD data for the triplicate simulations were averaged. Root-mean-square fluctuations (RMSF) of the peptide and biotin residues were analyzed and averaged for all three repetitions. The $\varphi / \psi$ dihedral combinations of the peptides were calculated and represented in Ramachandran plots for the most dominant structure present during the last $10 \mathrm{~ns}$ simulation time. The secondary structure content of the oligomeric protofibril systems (for main chain atoms) was analyzed for the last $10 \mathrm{~ns}$ simulation time by using the DSSP tool (Define Secondary Structure of Proteins) that was developed by Kabsch and Sander. ${ }^{46,47}$ Hydrophobic and hydrophilic solvent accessible surface areas (SASA) and solvation free energies per area were calculated for all systems for the last $10 \mathrm{~ns}$ of simulation time. ${ }^{48,49}$ Averages and standard errors were calculated for all parameters derived from the MD simulations.

Theoretical Prediction of the Solubility and Aggregation Propensities of GNNFGAIL Variants. The aggregation propensities of the GNNFGAIL variants (without Btn) were theoretically predicted by using $\mathrm{CamSol}^{50,51}$ (www-vendruscolo.ch.cam.ac.uk/ camsolmethod.html) and AGGRESCAN ${ }^{52}$ (bioinf.uab.es/aggrescan/ ). The biotin modification (Btn) is not parametrized in these algorithms. CamSol is a computational method that is based on the physicochemical properties of amino acids that are responsible for peptide solubility in water (e.g., hydrophobicity and electrostatic interactions). Intrinsic residue solubility profiles were determined based on the individual amino acids with respect to a window of neighboring residues. The CamSol intrinsic solubility score was calculated from residue solubility profiles. The AGGRESCAN algorithm is based on an aggregation-propensity scale for all natural amino acids. Aggregation profiles (a4v) are determined as window average of the amino acid aggregation-propensity values (a3v) of individual amino acids to account for the neighboring residues as well.

\section{RESULTS AND DISCUSSION}

Fibril Stability of Biotinylated GNNFGAIL. A peptide system with high aggregation propensity and a biotin anchor for conjugation of the fibrils to other structural elements based on the biotin-streptavidin system was established. Molecular dynamics (MD) simulations were performed to obtain information about the stability and the propensity to form structural fibril motifs in substituted peptides. The study involved the wild-type (wt) peptide NNFGAIL, GNNFGAIL (considering the N-terminal S20G substitution), and BtnGNNFGAIL. In addition, the NNGGAGL peptide was studied to validate our method, as fibrils of this variant were previously shown to be unstable in MD simulations. ${ }^{53}$ The MD starting structures consisted of five-stranded protofibrils with two $\beta$-sheets $(5 \times 2)$. To monitor the stability or disassembly of the modeled peptide oligomer structures, the systems were simulated for $100 \mathrm{~ns}$, starting with the known fibril structure of NNFGAIL for all peptide variants. The most dominant structures of the NNFGAIL, NNGGAGL, GNNFGAIL, and Btn-GNNFGAIL peptides during the last $10 \mathrm{~ns}$ of simulation time are shown in Figure 2a. The protofibril structure of NNGGAGL was significantly disturbed and not stable, as previously reported. ${ }^{53}$ NNFGAIL, GNNFGAIL, and BtnGNNFGAIL had a higher fibril stability. The root-meansquare deviations (RMSD) of the peptide backbones and the root-mean-square fluctuations (RMSF) of the peptide residues were calculated to quantify the stability or disassembly of peptide oligomer structures (Figure 2b). ${ }^{53-55}$ The NNGGAGL protofibril had the lowest stability, confirming the importance of the hydrophobic side chains phenylalanine (F23) and isoleucine (I26) for peptide fibril formation. ${ }^{53}$ The extension of the wild-type sequence (GNNFGAIL and BtnGNNFGAIL) enhanced the peptide fibril stability. In fact, Btn- 
a) Most dominant structures during the last $10 \mathrm{~ns}$ of simulation time

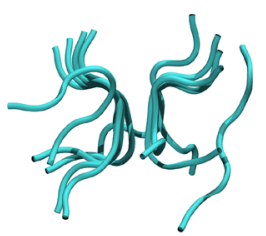

NNFGAIL

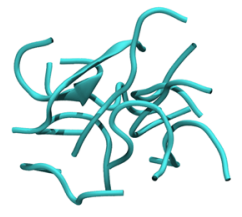

NNGGAGL

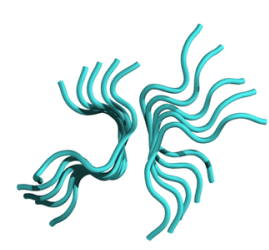

GNNFGAIL

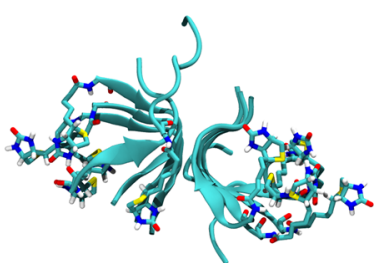

Btn-GNNFGAIL b) RMSD and RMSF analysis

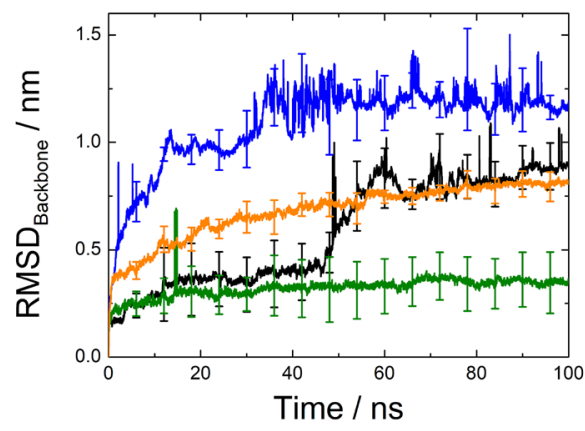

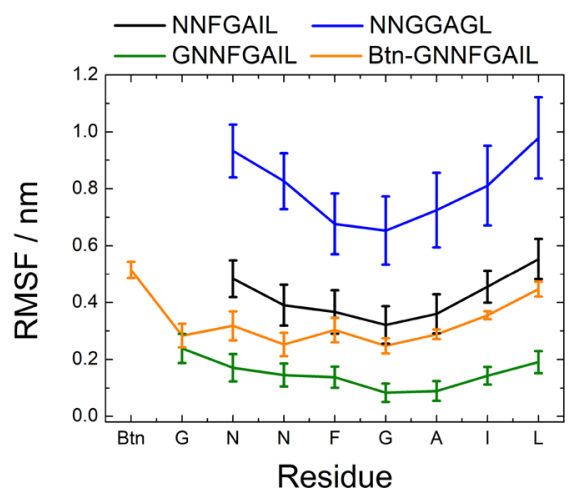

Figure 2. (a) The most dominant structures of the last $10 \mathrm{~ns}$ of simulation time (of all three repetitions each) are shown with the peptides in cartoon representation and Btn-G in licorice representation. Clustering analysis was performed, and the central structure of the largest cluster was determined. (b) Root-mean-square deviations (RMSD) for the peptide backbone and root-mean-square fluctuations (RMSF) of all peptide residues and biotin were calculated over the simulation time of $100 \mathrm{~ns}$. Standard errors of the mean are shown.

GNNFGAIL had final RMSD and RMSF deviations similar to NNFGAIL. However, the deviations for Btn-GNNFGAIL at the start of the simulations resulted from an optimization of the protofibril structure since the starting configuration of BtnGNNFGAIL was assumed as for NNFGAIL. In contrast, the deviations for NNFGAIL after around 50 ns were caused by a partial disintegration of the fibril structure. The GNNFGAIL protofibril was similarly stable as for Btn-GNNFGAIL; it did not need to optimize its structure as the addition of glycine (G) did not lead to larger structural changes.

Ramachandran plots of the most dominant peptide structures during the last $10 \mathrm{~ns}$ simulation time show a loss of the characteristic $\beta$-sheet structure of NNGGAGL whereas both GNNFGAIL and Btn-GNNFGAIL present an enhancement compared to NNFGAIL. The Ramachandran plots and the $\beta$-sheet and $\beta$-bridge secondary structure content of the last $10 \mathrm{~ns}$ of simulation time are shown in Figure 3. The highest $\beta$-sheet content was observed for Btn-GNNFGAIL. The MD simulations thus revealed that the $\mathrm{N}$-terminal modification of NNFGAIL with Btn-G had a positive impact on the stability of the peptide fibrils. Btn-GNNFGAIL also had the highest hydrophobic surface accessible surface area (SASA) and solvation free energy per area, indicating its high aggregation propensity (Supporting Information Figure S1).

Experimental studies on the fibrillation kinetics of NNFGAIL, GNNFGAIL, and Btn-GNNFGAIL based on thioflavin $\mathrm{T}$ (ThT) fluorescence confirmed the simulation results (Figure 4). ThT fluorescence assays are based on changes in the fluorescence properties of the ThT dye upon its binding to $\beta$-sheet-rich fibrils. ${ }^{56,57}$ Btn-GNNFGAIL aggregated much faster than NNFGAIL and GNNFGAIL. GNNFGAIL at first seemed stable in the simulations; however, it had a lower $\beta$-sheet content, hydrophobic domain, and solvation free energy per area than the wild-type NNFGAIL (see Figure 3 and Figure S1). Thus, GNNFGAIL fibrils might be stable once formed, but a similar or lower aggregation propensity as for NNFGAIL is expected. Although its aggregation kinetics were slower, typical amyloid fibrils were also found for NNFGAIL when studied by electron microscopy (SEM; see Figure S2). This is likely due to the SEM sample preparation, as the peptide solution was air-dried on the substrate which increased the peptide concentration and thus accelerated fibril growth.

Fibril Formation Kinetics of Alanine Scanning Variants of Btn-GNNFGAIL. To understand the role of single amino acids within Btn-GNNFGAIL for fibril growth and morphology, alanine scanning of the Btn-GNNFGAIL peptide was carried out. Compared to glycine and proline substitutions ( $\beta$-sheet breaker), it is assumed that alanine scanning has a reduced impact on the peptide backbone, which is important for NNFGAIL-derived peptides since their fibrillation is promoted by backbone-backbone interactions of the individual strands (Figure 1B). ${ }^{23}$ The kinetics of amyloid fibril formation was followed using ThT binding assays (Figure 4). ${ }^{56,57}$ The data show the fluorescence intensity after several time intervals under identical ambient conditions for each peptide (temperature and shaking intensity). Both the fluorescence intensities and the lag times for fibril formation vary between the peptide variants. This indicates that the sidechain functionalities in the $\operatorname{IAPP}_{21-27}$ peptide have a distinct influence on the nucleation and elongation phase of peptide aggregation. Considering the value after 1 day of incubation (green bars), the peptide variants Btn-GNN(F23A)GAIL, BtnGNNFGA(I26A)L, Btn-GNNFGAI(L27A), and Btn-GN(N22A)FGAIL had a reduced fluorescence intensity compared to the other peptides. This suggests that the residues F23, I26, L27, and N22 are important for the formation of $\beta$-sheet-rich nuclei that seed fibril formation. Once $\beta$-sheet-rich structures were formed for Btn-GN(N22A)FGAIL and Btn-GNN(F23A)GAIL after a lag time of several days, those peptide variants also aggregated. While Btn-GN(N22A)FGAIL rapidly 
a)

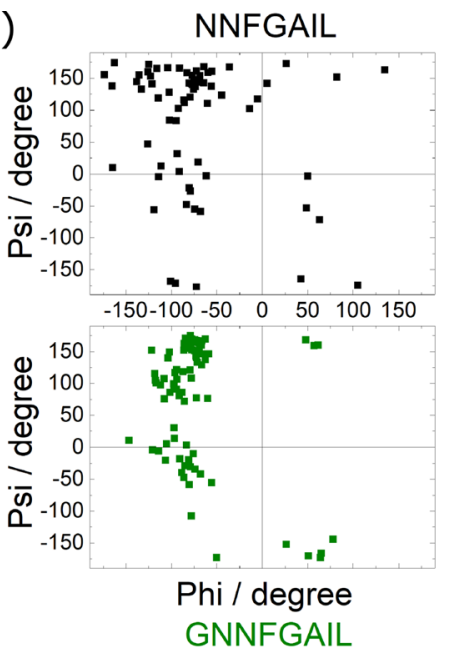

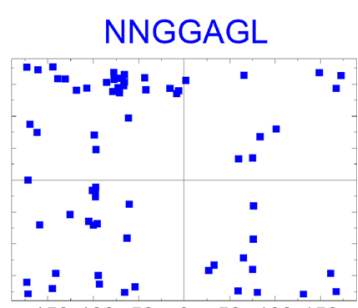

$-150-100-50 \quad 0 \quad 50 \quad 100 \quad 150$

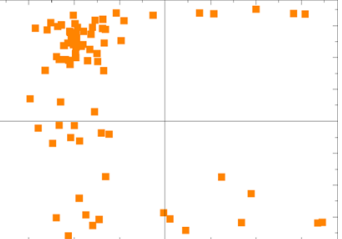

Phi / degree

Btn-GNNFGAIL

b)

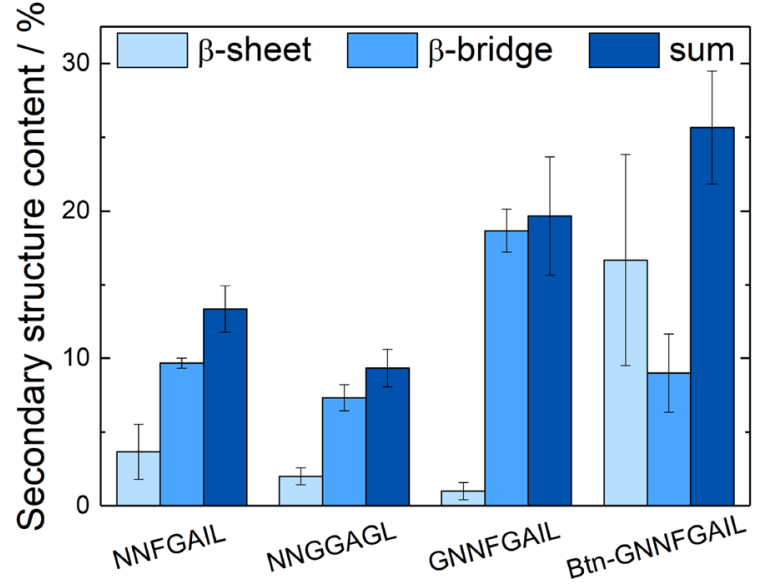

Figure 3. (a) Ramachandran plots for the most dominant structures (as shown in Figure $2 \mathrm{a}$ ) and (b) overview of $\beta$-sheet and $\beta$-bridge secondary structure content $($ sum $=\beta$-sheet $+\beta$-bridge $)$ during the last $10 \mathrm{~ns}$ of simulation time according to the DSSP algorithm. Negative phi and positive psi angles are characteristic for $\beta$-sheet structures. Larger parts of the peptide structures were identified as random coil. Standard errors of the mean are shown.

formed mature fibrils once the nuclei were present, BtnGNN(F23A)GAIL elongation was retarded, too. After one month of incubation, significant fluorescence signals were found in all samples, except for Btn-GNNFGA(I26A)L. The longest lag times of fibrillation were observed for the variants Btn-GNN(F23A)GAIL and Btn-GNNFGAI(L27A).

Morphologies of Alanine Scanning Variants of BtnGNNFGAIL. The peptide fibril morphologies after 1 and 17 days were studied by SEM (Figure 5 and Figure S3). After 1 day, large amounts of fibrils were formed for almost all alanine scanning variants, when dried on silicon wafers, with the exception of Btn-GN(N22A)FGAIL, Btn-GNN(F23A)GAIL, and Btn-GNNFGAI(L27A). In these cases, mostly amorphous peptide aggregates cover the surface of the silicon wafers, which have a tendency to arrange in a more or less nonordered phase. Btn-(G20A)NNFGAIL and Btn-G(N21A)NFGAIL formed thinner fibril structures, while Btn-GNNF(G24A)AIL, Btn-GNNFGAIL, and Btn-GNNFGA(I26A)L formed thicker ones. The morphologies of the supramolecular structures differ from mature NNFGAIL fibrils. NNFGAIL forms extremely dense fibril associates with a crystal-like superlattice and a high degree of stiffness (Figure 5 and Figure S2). In contrast, single

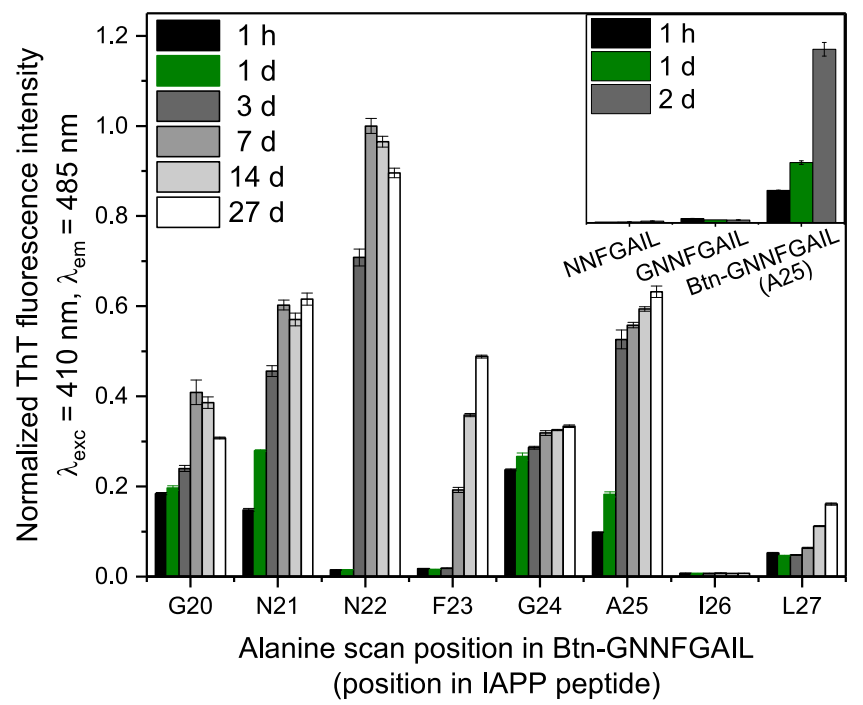

Figure 4. Comparison of the fibrillation kinetics of the wild-type NNFGAIL, GNNFGAIL, and Btn-GNNFGAIL and its alanine scanning variants using ThT fluorescence spectroscopy ([peptide] in the fibrillation assay $=1 \mathrm{mg} / \mathrm{mL}$ ). Standard errors of the mean are shown.

fibrils of Btn-(G20A)NNFGAIL are arranged in flexible ringshaped structures, which can be compressed to tight gels (Figure 5 and Figure S3). The stiffness appears dramatically reduced. The same feature was ultimately found for the BtnGNNFGAIL, indicating that the N-terminal modification has a high impact on the morphology of the fibrils, which is likely based on their intrinsic flexibility. After 17 days, the morphology slightly changed for the peptide Btn-G(N21A)NFGAIL which formed needle-like fibrils, which was not obvious after 1 day. A much higher amount of fibrils was observed for Btn-GNNFGA(I26A)L after 17 days with a comparable morphology to Btn-(G20A)NNFGAIL, BtnGNNFGAIL, and Btn-GNNF(G24A)AIL (Figure S3). The double variant Btn-GNN(F23A)GA(I26A)L formed no fibrils, even after 17 days. For Btn-GNNFGA(I26A)L, no fibrils were found in the ThT fluorescence assay after 1 day, while SEM revealed some fibrillar structures. This is likely caused by surface concentration effects during sample preparation for SEM. While the peptide containing droplets air-dried on the silicon supports, the peptide concentration increased, which increased the propensity for peptide aggregation.

Our results indicate that the substitution of F23A has a large negative impact on the formation of mature fibrils. Thus, F23 is a key residue for the aggregation of Btn-GNNFGAIL. This result is consistent with previously reported MD simulations, ${ }^{53}$ where glycine scanning variants of the non-biotinylated wildtype NNFGAIL were studied to understand the effect of single-point substitutions on the amyloidogenic propensity in terms of the sheet-to-sheet and strand-to-strand interactions. In agreement with our experimental results, the variants NN(F23G)GAIL and NNFGA(I26G)L had a lower fibril stability followed by (N21G)NFGAIL and N(N22G)FGAIL. Our findings also agree with previous observations when IAPP (segment) mutations were investigated. For instance, the variants $\mathrm{N}(\mathrm{F} 23 \mathrm{Y})$ GAILSS, SN(N22P)FGAILSS, SNNFGA(I26P)LSS, and SNNFGAI(L27P) aggregated to a lower extent. $^{58,59}$ No or a reduced amyloid fibril formation was observed under experimental conditions when phenylalanine 


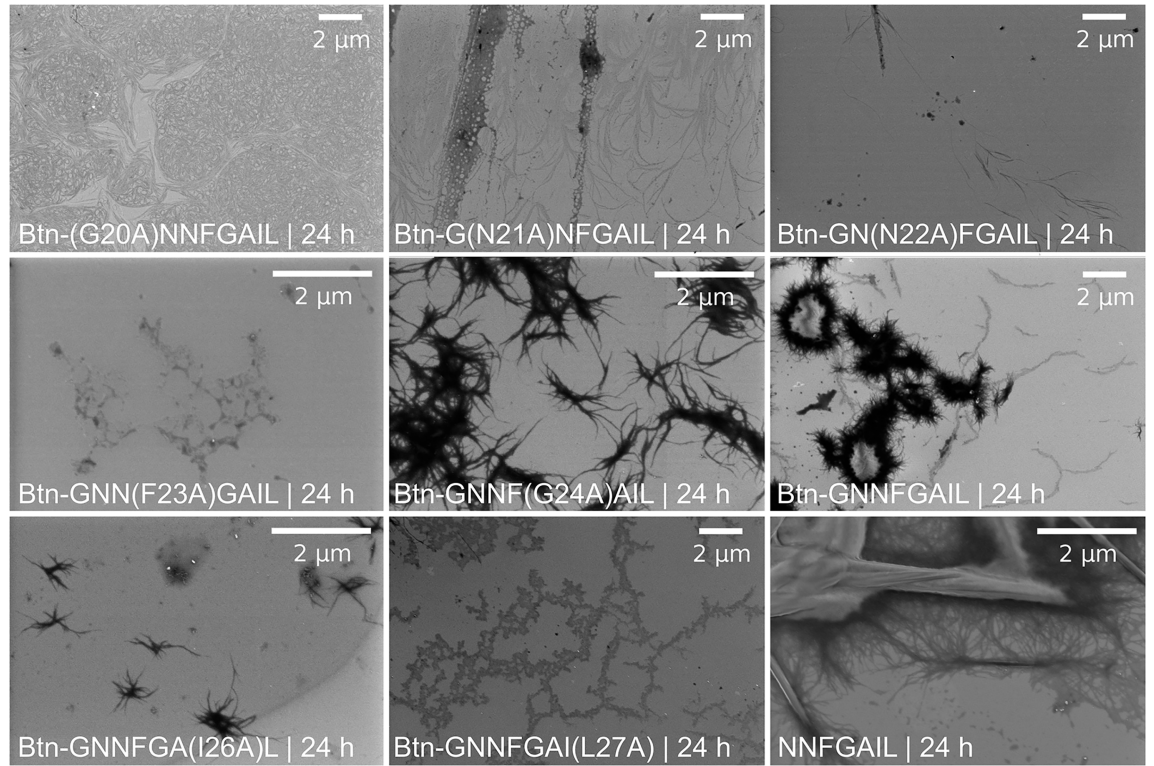

Figure 5. SEM images of fibrils of the alanine scanning peptides of Btn-GNNFGAIL after $24 \mathrm{~h}$ ([peptide] in the fibrillation assay $=0.1 \mathrm{mg} / \mathrm{mL}$ ).

a) CamSol

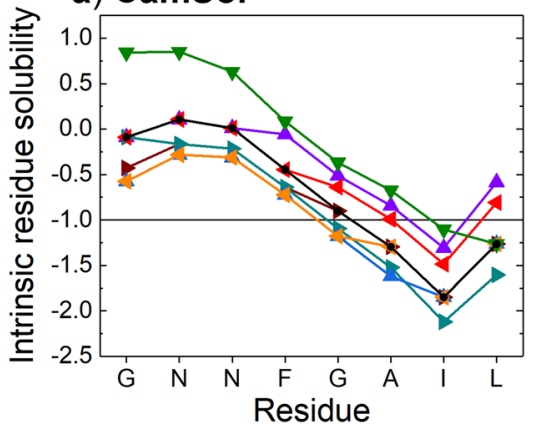

b) AGGRESCAN

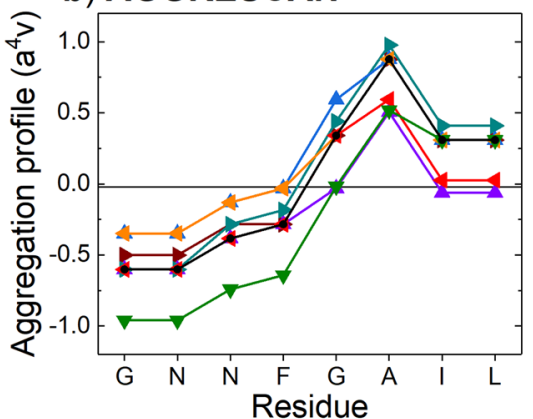

c) Comparison of exp. and predicted data

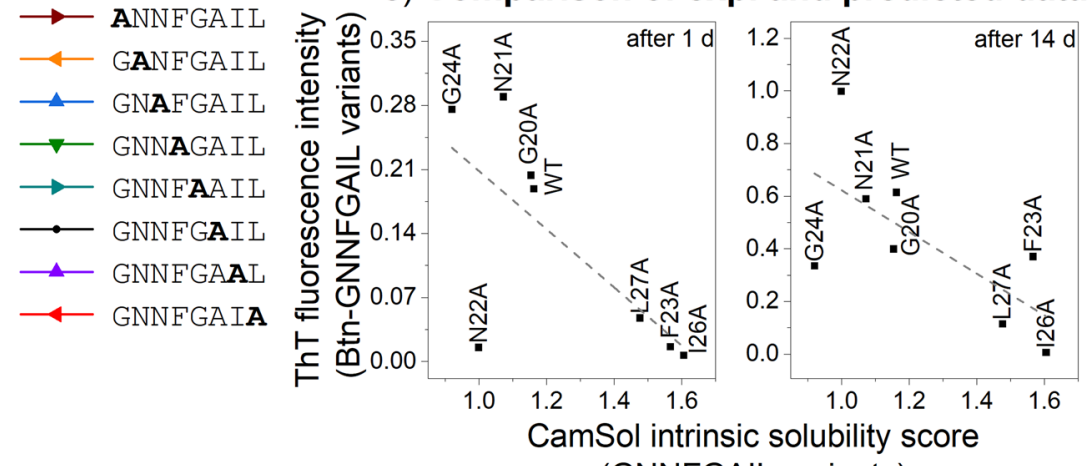

(GNNFGAIL variants)

Figure 6. Differences in the aggregation kinetics of the Btn-GNNFGAIL variants in the ThT fluorescence assay were compared to theoretical solubility and aggregation prediction models of GNNFGAIL. (a) CamSol intrinsic residue solubilities of the GNNFGAIL variants. ${ }^{50,51}$ Low values indicate a low solubility and possibly high aggregation propensity. Regions below -1 have a very low solubility. (b) AGGRESCAN aggregation profiles of the GNNFGAIL variants. ${ }^{52}$ Higher values (above -0.02) indicate regions of high aggregation propensity. (c) Comparison of experimental ThT fluorescence intensities of Btn-GNNFGAIL variants after 1 and 14 days with the CamSol intrinsic solubility scores of the GNNFGAIL variants. ${ }^{50,51}$ Overall, low CamSol intrinsic solubility scores related to a large aggregation in the kinetic assay. An exception was GN(N22A)FGAIL after 1 day, likely due to a kinetic effect (longer lag time). Gray dashed lines are used to guide the reader's eye (based on linear fits of data); however, no mathematical correlation is intended.

was replaced with alanine in full-length IAPP and selected peptide segments. ${ }^{60}$ A summary on the influence of amino acid substitutions in IAPP and derived peptides from previous studies is provided in Table S2, emphasizing the role of each residue.
Theoretical Predictions. Theoretical predictions based on the contribution of each amino acid in GNNFGAIL to the overall solubility and propensity to form fibrillar aggregates revealed that the F23A variant had the largest impact, followed by I26A and L27A (Figure 6). ${ }^{50-52}$ These calculations are in 
agreement with the experimental results obtained in the ThT kinetic assay and SEM measurements for Btn-GNNFGAIL, confirming the high relevance of the phenylalanine, isoleucine and leucine residues for peptide fibril formation, especially its early nucleation phase. It needs to be noted that the predictions were made for GNNFGAIL variants since the biotin modification (Btn) is not parametrized in these algorithms. Thus, it was assumed that biotin has the same effect on solubility in each peptide variant. This assumption might have led to deviations in the trend of the predictions. A lower solubility and higher aggregation propensity were found for the G20A, N21A, N22A, and G24A variants compared to the unmodified GNNFGAIL peptide, in agreement with the ThT assay (Figure 4). One outlier between the predicted solubility for GNNFGAIL variants and the experimentally observed amyloid formation for Btn-GNNFGAIL after 1 day is the $\mathrm{N} 22 \mathrm{~A}$ variant. Although this variant is poorly soluble, it did not form many fibrils. This could be likely due to the biotinylation or a kinetic effect, increasing the lag time for the formation of a critical nucleus, until large amounts of fibrils were formed after 3 days (Figures 4 and 6).

The substitution of phenylalanine in the variant BtnGNN(F23A)GAIL had a negative impact on the formation of fibrils. Considering the arrangement of amino acid side chains from the X-ray structure, strand-to-strand hydrophobic interactions of phenylalanine-isoleucine are the main interaction modes (see Figure 1B). ${ }^{23}$ The substitution of phenylalanine with the smaller and less hydrophobic alanine (or glycine) reduced these attractive interactions, independent of the incorporation of biotin into the peptide. The importance of hydrophobic residues and $\pi$-stacking for amyloid fibril formation has previously been reported. ${ }^{60-62}$ Contrarily, alanine substitutions at positions $\mathrm{N} 21$ and to a lesser extent G24, considering steric restrictions from the X-ray structure, had only a minor impact on the fibrillation process, and therefore these sites are suitable candidates for further substitution and modification processes. However, the impact of modifications on peptide fibrillation will also depend on the size and structure of the incorporated groups. To study biologically relevant peptides or proteins, label-free approaches should be favored to reproduce the original fibril kinetics and morphologies.

Immobilization to Streptavidin-Coated Surfaces. Fibril-modified surfaces are of interest for different purposes, for instance, their usage as a new class of morphologically and chemically engineered biomaterials, which may be involved in fibril-cell or fibril-nanoparticle interactions. ${ }^{63-68}$ Because the amount of biological sample materials is limited, microfluidic approaches are readily advantageous over conventional laboratory techniques. ${ }^{69}$ The spatially controlled immobilization of fibrils to surfaces is the first step toward their integration into multifunctional microfluidic systems. A streptavidin-coated glass slide was used as a model to demonstrate the spatial immobilization of biotinylated fibrils to surfaces as a first proof-of-concept, however, even other binding concepts may be suitable. To achieve the goal, the streptavidin-coated slide was exposed to an atmospheric oxygen plasma through a mask allowing for streptavidin inactivation and degradation at the exposed areas. Different surface modification techniques, such as laser structuring, could also be applied at this stage. ${ }^{70}$ After treatment, exposed and unexposed areas were incubated with a peptide solution of Btn-GNNFGAIL under fibrillation conditions (Figure 1D).
The spatial resolution for fibril immobilization on the surface by using this approach is limited by the resolution of the streptavidin structure on the glass slide obtained during the oxygen plasma treatment.

SEM revealed a higher degree of immobilized fibrils in the streptavidin-coated areas, whereas plasma-cleaned areas showed a much lower degree of bound fibrils (Figure 7 and Figure S4). Image analysis (Matlab R2018b, Gwyddion 2.52 ${ }^{71}$ ) revealed a fibril surface coverage of at least $24 \%$ on the streptavidin-coated (untreated) area and 5\% fibril coverage on the plasma-treated side (see Figure S5 for masked images). Instead of a homogeneous surface coverage, the fibrils formed islets of a few tens of micrometers (Figure 7c). Smaller and thinner fibrils can be seen in the areas in between these larger fibril aggregates. While the image analysis only considered the larger fibril aggregates, the results demonstrate that amyloid fibrils were spatially controlled immobilized by using the biotin-streptavidin interaction.

The herein-presented approach is highly compatible with our recently developed method of inverse microfluidic printing

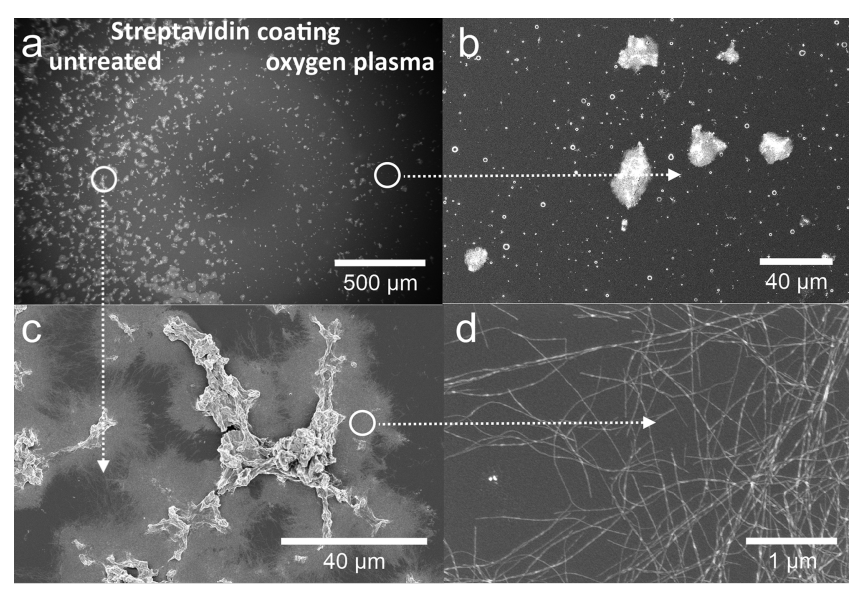

Figure 7. SEM images of spatially immobilized fibrils of BtnGNNFGAIL on a streptavidin-coated glass slide. (a) While the left side of the streptavidin-coated glass slide was untreated, the right side was cleaned under an oxygen plasma. Amyloid fibrils were largely immobilized in the streptavidin-coated area. (b) Typical SEM image of an area that was oxygen plasma treated with not many immobilized peptide fibrils. (c) Typical SEM image of a streptavidin-coated (untreated) area with many immobilized peptide fibrils. (d) Magnified area of the bound amyloid fibrils showing the typical fibril structure.

(cryoprinting). ${ }^{72}$ Cryoprinting enables the fabrication of microfluidic systems on a time scale of a few minutes by aqueous printing and freezing of microstructures on glass slides by using the drop-on-demand technology, which are subsequently encapsulated in a photocured polymer within milliseconds. Once the ice template melts, a microfluidic polymer channel is obtained on the glass slide. Fibrillar nanostructures and their gels could thus be integrated into such microfluidic systems under spatial control in the near future. The immobilized fibrils on the glass slide could serve as affinity materials ${ }^{73,74}$ or templates for electrode materials 6 within the microchannel that was formed.

\section{CONCLUSIONS}

To obtain insight into the contribution of single amino acids on the fibrillation of Btn-GNNFGAIL peptide (Btn-G- 
$\mathrm{IAPP}_{21-27}$ ), we applied experimental alanine scanning combined with computational methods. We identified phenylalanine (F23), leucine (L27), and isoleucine (I26) as key residues emphasizing the role of aromatic/hydrophobic interactions for fibrillation, which is in agreement with previous studies of the non-biotinylated peptide. Asparagine (N21) and glycine (G24) played a minor role for fibril formation and are thus potential targets for further peptide modifications. We have demonstrated the spatially controlled immobilization of such fibrils on surfaces as a proof-of-concept for their integration into microfluidic systems.

\section{ASSOCIATED CONTENT}

\section{(s) Supporting Information}

The Supporting Information is available free of charge at https://pubs.acs.org/doi/10.1021/acs.biomac.9b01466.

MALDI-TOF-MS peptide characterization; experimental section for atmospheric oxygen plasma treatment; surface-accessible-surface areas of peptide protofibrils from MD simulations; SEM images of NNFGAIL-fibrils; SEM images of fibrils obtained from the alanine scanning peptides of Btn-GNNFGAIL after 17 days; summary of results and previous studies on the impact of amino acid substitutions in IAPP segments on peptide aggregation; SEM images of spatially immobilized fibrils of Btn-GNNFGAIL on streptavidin-coated glass slides (PDF)

\section{AUTHOR INFORMATION}

\section{Corresponding Author}

Bernd Abel - Leibniz-Institute of Surface Engineering (IOM), Leipzig, Germany, and Leipzig University, Leipzig, Germany; ○ orcid.org/0000-0001-6032-1680; Email: bernd.abel@uni-leipzig.de

\section{Other Authors}

Torsten John - Leibniz-Institute of Surface Engineering (IOM), Leipzig, Germany, and Leipzig University,

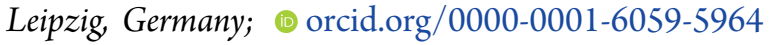

Juhaina Bandak - Leibniz-Institute of Surface Engineering (IOM), Leipzig, Germany

Nilushiya Sarveson - Leibniz-Institute of Surface Engineering (IOM), Leipzig, Germany

Claudia Hackl - Leibniz-Institute of Surface Engineering (IOM), Leipzig, Germany

Herre Jelger Risselada - Leibniz-Institute of Surface Engineering (IOM), Leipzig, Germany, and GeorgAugust-Universität Göttingen, Göttingen, Germany

Andrea Prager - Leibniz-Institute of Surface Engineering (IOM), Leipzig, Germany

Christian Elsner - Leibniz-Institute of Surface Engineering (IOM), Leipzig, Germany

Complete contact information is available at: https://pubs.acs.org/10.1021/acs.biomac.9b01466

\section{Author Contributions}

The study was designed by C.E., T.J., and B.A. ThT experiments were performed and analyzed by N.S., J.B., and C.E. SEM measurements were performed by A.P. Surface immobilization was done by C.E. and N.S. Peptides were synthesized by J.B. and C.E. Computer simulations and theoretical predictions were planned, performed, and analyzed by T.J. Mass spectrometry was performed by J.B. and C.H. The experimental and theoretical results were interpreted by C.E., T.J., N.S., H.J.R., and B.A. The manuscript was written by C.E. and T.J. All authors have given approval to the final version of the manuscript.

\section{Funding}

This research was financially supported by the Deutsche Forschungsgemeinschaft (DFG, German Research Foundation, Project 189853844, SFB-TRR 102, B1). T.J. thanks the Friedrich-Ebert-Stiftung for a $\mathrm{PhD}$ fellowship.

\section{Notes}

The authors declare no competing financial interest.

\section{REFERENCES}

(1) Ke, P. C.; Sani, M. A.; Ding, F.; Kakinen, A.; Javed, I.; Separovic, F.; Davis, T. P.; Mezzenga, R. Implications of Peptide Assemblies in Amyloid Diseases. Chem. Soc. Rev. 2017, 46 (21), 6492-6531.

(2) Chiti, F.; Dobson, C. M. Protein Misfolding, Amyloid Formation, and Human Disease: A Summary of Progress Over the Last Decade. Annu. Rev. Biochem. 2017, 86 (1), 27-68.

(3) Wei, G.; Su, Z.; Reynolds, N. P.; Arosio, P.; Hamley, I. W.; Gazit, E.; Mezzenga, R. Self-Assembling Peptide and Protein Amyloids: From Structure to Tailored Function in Nanotechnology. Chem. Soc. Rev. 2017, 46 (15), 4661-4708.

(4) Hauser, C. A. E.; Maurer-Stroh, S.; Martins, I. C. Amyloid-Based Nanosensors and Nanodevices. Chem. Soc. Rev. 2014, 43 (15), 53265345.

(5) Feng, Y.; Wang, H.; Zhang, J.; Song, Y.; Meng, M.; Mi, J.; Yin, H.; Liu, L. Bio-Inspired Synthesis of Au Nanostructures Templated from Amyloid $\beta$ Peptide Assembly with Enhanced Catalytic Activity. Biomacromolecules 2018, 19, 2432-2442.

(6) Bandak, J.; Petzold, J.; Hatahet, H.; Prager, A.; Kersting, B.; Elsner, C.; Abel, B. Interconnected Electrocatalytic Pt-Metal Networks by Plasma Treatment of Nanoparticle-Peptide Fibril Assemblies. RSC Adv. 2019, 9 (10), 5558-5569.

(7) Elsner, C.; Hintzen, D.; Prager, A.; Siefermann, K. R.; Abel, B. Supramolecular Assembly of Functional Hybrid Fibrils with Peptide$\pi$-System-Peptide Monomers Near Silver-Nanoparticles. Z. Phys. Chem. 2015, 229 (3), 427-442.

(8) Garvey, M.; Gras, S. L.; Meehan, S.; Meade, S. J.; Carver, J. A.; Gerrard, J. A. Protein Nanofibres of Defined Morphology Prepared from Mixtures of Crude Crystallins. Int. J. Nanotechnol. 2009, 6 (34), 258-273.

(9) Li, D.; Jones, E. M.; Sawaya, M. R.; Furukawa, H.; Luo, F.; Ivanova, M.; Sievers, S. A.; Wang, W.; Yaghi, O. M.; Liu, C.; Eisenberg, D. S. Structure-Based Design of Functional Amyloid Materials. J. Am. Chem. Soc. 2014, 136 (52), 18044-18051.

(10) Norlin, N.; Hellberg, M.; Filippov, A.; Sousa, A. A.; Gröbner, G.; Leapman, R. D.; Almqvist, N.; Antzutkin, O. N. Aggregation and Fibril Morphology of the Arctic Mutation of Alzheimer's A $\beta$ Peptide by CD, TEM, STEM and in Situ AFM. J. Struct. Biol. 2012, 180 (1), 174-189.

(11) Mezzenga, R.; Fischer, P. The Self-Assembly, Aggregation and Phase Transitions of Food Protein Systems in One, Two and Three Dimensions. Rep. Prog. Phys. 2013, 76 (4), 046601.

(12) Adamcik, J.; Mezzenga, R. Proteins Fibrils from a Polymer Physics Perspective. Macromolecules 2012, 45 (3), 1137-1150.

(13) Xue, W. F.; Hellewell, A. L.; Gosal, W. S.; Homans, S. W.; Hewitt, E. W.; Radford, S. E. Fibril Fragmentation Enhances Amyloid Cytotoxicity. J. Biol. Chem. 2009, 284 (49), 34272-34282.

(14) Stefani, M. Biochemical and Biophysical Features of Both Oligomer/Fibril and Cell Membrane in Amyloid Cytotoxicity. FEBS J. 2010, 277 (22), 4602-4613. 
(15) Knowles, T. P. J.; Buehler, M. J. Nanomechanics of Functional and Pathological Amyloid Materials. Nat. Nanotechnol. 2011, 6 (8), 469-479.

(16) Wang, S.-T.; Lin, Y.; Spencer, R. K.; Thomas, M. R.; Nguyen, A. I.; Amdursky, N.; Pashuck, E. T.; Skaalure, S. C.; Song, C. Y.; Parmar, P. A.; Morgan, R. M.; Ercius, P.; Aloni, S.; Zuckermann, R. N.; Stevens, M. M. Sequence-Dependent Self-Assembly and Structural Diversity of Islet Amyloid Polypeptide-Derived $\beta$-Sheet Fibrils. ACS Nano 2017, 11 (9), 8579-8589.

(17) Tycko, R. Physical and Structural Basis for Polymorphism in Amyloid Fibrils. Protein Sci. 2014, 23 (11), 1528-1539.

(18) Domigan, L. J.; Healy, J. P.; Meade, S. J.; Blaikie, R. J.; Gerrard, J. A. Controlling the Dimensions of Amyloid Fibrils: Toward Homogenous Components for Bionanotechnology. Biopolymers 2012, 97 (2), 123-133.

(19) Waldauer, S. A.; Hassan, S.; Paoli, B.; Donaldson, P. M.; Pfister, R.; Hamm, P.; Caflisch, A.; Pellarin, R. Photocontrol of Reversible Amyloid Formation with a Minimal-Design Peptide. J. Phys. Chem. B 2012, 116 (30), 8961-8973.

(20) Deeg, A. A.; Schrader, T. E.; Kempter, S.; Pfizer, J.; Moroder, L.; Zinth, W. Light-Triggered Aggregation and Disassembly of Amyloid-like Structures. ChemPhysChem 2011, 12 (3), 559-562.

(21) Cao, P.; Abedini, A.; Wang, H.; Tu, L. H.; Zhang, X.; Schmidt, A. M.; Raleigh, D. P. Islet Amyloid Polypeptide Toxicity and Membrane Interactions. Proc. Natl. Acad. Sci. U. S. A. 2013, 110 (48), 19279-19284.

(22) Brender, J. R.; Salamekh, S.; Ramamoorthy, A. Membrane Disruption and Early Events in the Aggregation of the Diabetes Related Peptide IAPP from a Molecular Perspective. Acc. Chem. Res. 2012, 45 (3), 454-462.

(23) Wiltzius, J. J. W.; Sievers, S. A.; Sawaya, M. R.; Cascio, D.; Popov, D.; Riekel, C.; Eisenberg, D. Atomic Structure of the Cross- $\beta$ Spine of Islet Amyloid Polypeptide (Amylin). Protein Sci. 2008, 17 (9), 1467-1474.

(24) Morrison, K. L.; Weiss, G. A. Combinatorial Alanine-Scanning. Curr. Opin. Chem. Biol. 2001, 5 (3), 302-307.

(25) Stayton, P. S.; Freitag, S.; Klumb, L. A.; Chilkoti, A.; Chu, V.; Penzotti, J. E.; To, R.; Hyre, D.; Le Trong, I.; Lybrand, T. P.; Stenkamp, R. E. Streptavidin-Biotin Binding Energetics. Biomol. Eng. 1999, 16 (1-4), 39-44.

(26) Diamandis, E. P.; Christopoulos, T. K. The Biotin-(Strept)Avidin System: Principles and Applications in Biotechnology. Clin. Chem. 1991, 37 (5), 625-636.

(27) Seino, S. S20G Mutation of the Amylin Gene Is Associated with Type II Diabetes in Japanese. Diabetologia 2001, 44 (7), 906-909.

(28) Sakagashira, S.; Hiddinga, H. J.; Tateishi, K.; Sanke, T.; Hanabusa, T.; Nanjo, K.; Eberhardt, N. L. S20G Mutant Amylin Exhibits Increased in Vitro Amyloidogenicity and Increased Intracellular Cytotoxicity Compared to Wild-Type Amylin. Am. J. Pathol. 2000, 157 (6), 2101-2109.

(29) Rose, A. S.; Bradley, A. R.; Valasatava, Y.; Duarte, J. M.; Prlic, A.; Rose, P. W. NGL Viewer: Web-Based Molecular Graphics for Large Complexes. Bioinformatics 2018, 34 (21), 3755-3758.

(30) Malde, A. K.; Zuo, L.; Breeze, M.; Stroet, M.; Poger, D.; Nair, P. C.; Oostenbrink, C.; Mark, A. E. An Automated Force Field Topology Builder (ATB) and Repository: Version 1.0. J. Chem. Theory Comput. 2011, 7, 4026-4037.

(31) Canzar, S.; El-Kebir, M.; Pool, R.; Elbassioni, K.; Malde, A. K.; Mark, A. E.; Geerke, D. P.; Stougie, L.; Klau, G. W. Charge Group Partitioning in Biomolecular Simulation. J. Comput. Biol. 2013, 20 (3), $188-198$.

(32) Koziara, K. B.; Stroet, M.; Malde, A. K.; Mark, A. E. Testing and Validation of the Automated Topology Builder (ATB) Version 2.0: Prediction of Hydration Free Enthalpies. J. Comput.-Aided Mol. Des. 2014, 28 (3), 221-233.

(33) Berendsen, H. J. C.; Postma, J. P. M.; van Gunsteren, W. F.; Hermans, J. Interaction Models for Water in Relation to Protein Hydration. In The Jerusalem Symposia on Quantum Chemistry and Biochemistry; Pullman, B., Ed.; Reidel: Dordrecht, 1981; pp 331-342.
(34) Hess, B.; Kutzner, C.; van der Spoel, D.; Lindahl, E. GROMACS 4: Algorithms for Highly Efficient, Load-Balanced, and Scalable Molecular Simulation. J. Chem. Theory Comput. 2008, 4, 435-447.

(35) van der Spoel, D.; Lindahl, E.; Hess, B.; Groenhof, G.; Mark, A. E.; Berendsen, H. J. C. GROMACS: Fast, Flexible, and Free. J. Comput. Chem. 2005, 26, 1701-1718.

(36) Lindahl, E.; Hess, B.; van der Spoel, D. GROMACS 3.0: A Package for Molecular Simulation and Trajectory Analysis. J. Mol. Model. 2001, 7, 306-317.

(37) Berendsen, H. J. C.; van der Spoel, D.; van Drunen, R. GROMACS: A Message-Passing Parallel Molecular Dynamics Implementation. Comput. Phys. Commun. 1995, 91, 43-56.

(38) Schmid, N.; Eichenberger, A. P.; Choutko, A.; Riniker, S.; Winger, M.; Mark, A. E.; van Gunsteren, W. F. Definition and Testing of the GROMOS Force-Field Versions 54A7 and 54B7. Eur. Biophys. J. 2011, 40, 843-856.

(39) Hess, B.; Bekker, H.; Berendsen, H. J. C.; Fraaije, J. G. E. M. LINCS: A Linear Constraint Solver for Molecular Simulations. J. Comput. Chem. 1997, 18, 1463-1472.

(40) Hess, B. P-LINCS: A Parallel Linear Constraint Solver for Molecular Simulation. J. Chem. Theory Comput. 2008, 4 (1), 116122.

(41) Miyamoto, S.; Kollman, P. A. SETTLE: An Analytical Version of the SHAKE and RATTLE Algorithm for Rigid Water Models. J. Comput. Chem. 1992, 13 (8), 952-962.

(42) Tironi, I. G.; Sperb, R.; Smith, P. E.; van Gunsteren, W. F. A Generalized Reaction Field Method for Molecular Dynamics Simulations. J. Chem. Phys. 1995, 102 (13), 5451-5459.

(43) Periole, X.; Rampioni, A.; Vendruscolo, M.; Mark, A. E. Factors That Affect the Degree of Twist in $\beta$-Sheet Structures: A Molecular Dynamics Simulation Study of a Cross- $\beta$ Filament of the GNNQQNY Peptide. J. Phys. Chem. B 2009, 113 (6), 1728-1737.

(44) Berendsen, H. J. C.; Postma, J. P. M.; van Gunsteren, W. F.; DiNola, A.; Haak, J. R. Molecular Dynamics with Coupling to an External Bath. J. Chem. Phys. 1984, 81, 3684-3690.

(45) Humphrey, W.; Dalke, A.; Schulten, K. VMD: Visual Molecular Dynamics. J. Mol. Graphics 1996, 14 (1), 33-38.

(46) Touw, W. G.; Baakman, C.; Black, J.; Te Beek, T. A. H.; Krieger, E.; Joosten, R. P.; Vriend, G. A Series of PDB-Related Databanks for Everyday Needs. Nucleic Acids Res. 2015, 43 (D1), D364-D368.

(47) Kabsch, W.; Sander, C. Dictionary of Protein Secondary Structure - Pattern-Recognition of Hydrogen-Bonded and Geometrical Features. Biopolymers 1983, 22 (12), 2577-2637.

(48) Eisenhaber, F.; Lijnzaad, P.; Argos, P.; Sander, C.; Scharf, M. The Double Cubic Lattice Method: Efficient Approaches to Numerical Integration of Surface Area and Volume and to Dot Surface Contouring of Molecular Assemblies. J. Comput. Chem. 1995, 16 (3), 273-284.

(49) Eisenberg, D.; McLachlan, A. D. Solvation Energy in Protein Folding and Binding. Nature 1986, 319 (6050), 199-203.

(50) Sormanni, P.; Aprile, F. A.; Vendruscolo, M. The CamSol Method of Rational Design of Protein Mutants with Enhanced Solubility. J. Mol. Biol. 2015, 427 (2), 478-490.

(51) Sormanni, P.; Amery, L.; Ekizoglou, S.; Vendruscolo, M.; Popovic, B. Rapid and Accurate in Silico Solubility Screening of a Monoclonal Antibody Library. Sci. Rep. 2017, 7 (1), 8200.

(52) Conchillo-Solé, O.; de Groot, N. S.; Avilés, F. X.; Vendrell, J.; Daura, X.; Ventura, S. AGGRESCAN: A Server for the Prediction and Evaluation of "Hot Spots" of Aggregation in Polypeptides. BMC Bioinf. 2007, 8 (1), 65

(53) Berhanu, W. M.; Masunov, A. E. Unique Example of Amyloid Aggregates Stabilized by Main Chain H-Bond Instead of the Steric Zipper: Molecular Dynamics Study of the Amyloidogenic Segment of Amylin Wild-Type and Mutants. J. Mol. Model. 2012, 18 (3), 891903.

(54) Zheng, J.; Jang, H.; Ma, B.; Tsai, C.-J.; Nussinov, R. Modeling the Alzheimer $\mathrm{A} \beta 17-42$ Fibril Architecture: Tight Intermolecular 
Sheet-Sheet Association and Intramolecular Hydrated Cavities. Biophys. J. 2007, 93 (9), 3046-3057.

(55) Buchete, N.-V.; Hummer, G. Structure and Dynamics of Parallel $\beta$-Sheets, Hydrophobic Core, and Loops in Alzheimer's $\mathrm{A} \beta$ Fibrils. Biophys. J. 2007, 92 (9), 3032-3039.

(56) Groenning, M. Binding Mode of Thioflavin $\mathrm{T}$ and Other Molecular Probes in the Context of Amyloid Fibrils-Current Status. J. Chem. Biol. 2010, 3 (1), 1-18.

(57) Khurana, R.; Coleman, C.; Ionescu-Zanetti, C.; Carter, S. A.; Krishna, V.; Grover, R. K.; Roy, R.; Singh, S. Mechanism of Thioflavin T Binding to Amyloid Fibrils. J. Struct. Biol. 2005, 151 (3), 229-238.

(58) Porat, Y.; Stepensky, A.; Ding, F. X.; Naider, F.; Gazit, E. Completely Different Amyloidogenic Potential of Nearly Identical Peptide Fragments. Biopolymers 2003, 69 (2), 161-164.

(59) Moriarty, D. F.; Raleigh, D. P. Effects of Sequential Proline Substitutions on Amyloid Formation by Human Amylin20-29. Biochemistry 1999, 38 (6), 1811-1818.

(60) Bakou, M.; Hille, K.; Kracklauer, M.; Spanopoulou, A.; Frost, C. V.; Malideli, E.; Yan, L. M.; Caporale, A.; Zacharias, M.; Kapurniotu, A. Key Aromatic/Hydrophobic Amino Acids Controlling a Cross-Amyloid Peptide Interaction versus Amyloid Self-Assembly. J. Biol. Chem. 2017, 292 (35), 14587-14602.

(61) Martin, L. L.; Kubeil, C.; Piantavigna, S.; Tikkoo, T.; Gray, N. P.; John, T.; Calabrese, A. N.; Liu, Y.; Hong, Y.; Hossain, M. A.; Patil, N.; Abel, B.; Hoffmann, R.; Bowie, J. H.; Carver, J. A. Amyloid Aggregation and Membrane Activity of the Antimicrobial Peptide Uperin 3.5. Pept. Sci. 2018, 110 (3), e24052.

(62) John, T.; Dealey, T. J. A.; Gray, N. P.; Patil, N. A.; Hossain, M. A.; Abel, B.; Carver, J. A.; Hong, Y.; Martin, L. L. The Kinetics of Amyloid Fibrillar Aggregation of Uperin 3.5 Is Directed by the Peptide's Secondary Structure. Biochemistry 2019, 58 (35), 36563668.

(63) Walsh, T. R.; Knecht, M. R. Biointerface Structural Effects on the Properties and Applications of Bioinspired Peptide-Based Nanomaterials. Chem. Rev. 2017, 117 (20), 12641-12704.

(64) Das, S.; Jacob, R. S.; Patel, K.; Singh, N.; Maji, S. K. Amyloid Fibrils: Versatile Biomaterials for Cell Adhesion and Tissue Engineering Applications. Biomacromolecules 2018, 19 (6), 18261839.

(65) Li, C.; Qin, R.; Liu, R.; Miao, S.; Yang, P. Functional Amyloid Materials at Surfaces/Interfaces. Biomater. Sci. 2018, 6 (3), 462-472.

(66) John, T.; Gladytz, A.; Kubeil, C.; Martin, L. L.; Risselada, H. J.; Abel, B. Impact of Nanoparticles on Amyloid Peptide and Protein Aggregation: A Review with a Focus on Gold Nanoparticles. Nanoscale 2018, 10 (45), 20894-20913.

(67) Pagel, M.; Hassert, R.; John, T.; Braun, K.; Wießler, M.; Abel, B.; Beck-Sickinger, A. G. Multifunctional Coating Improves Cell Adhesion on Titanium by Using Cooperatively Acting Peptides. Angew. Chem., Int. Ed. 2016, 55 (15), 4826-4830.

(68) Gladytz, A.; Abel, B.; Risselada, H. J. Gold-Induced Fibril Growth: The Mechanism of Surface-Facilitated Amyloid Aggregation. Angew. Chem., Int. Ed. 2016, 55 (37), 11242-11246.

(69) Herling, T. W.; Levin, A.; Saar, K. L.; Dobson, C. M.; Knowles, T. P. J. Microfluidic Approaches for Probing Amyloid Assembly and Behaviour. Lab Chip 2018, 18 (7), 999-1016.

(70) Zimmer, K.; Hirsch, D.; Bigl, F. Excimer Laser Machining for the Fabrication of Analogous Microstructures. Appl. Surf. Sci. 1996, 96-98, 425-429.

(71) Nečas, D.; Klapetek, P. Gwyddion: An Open-Source Software for SPM Data Analysis. Cent. Eur. J. Phys. 2012, 10 (1), 181-188.

(72) Elsner, C.; Abel, B.; Charvat, A. Verfahren zur Herstellung eines Mikrosystembauteils. Patent Application No. DE 102017130947.0 (A1), December 21, 2017.

(73) Gladytz, A.; John, T.; Gladytz, T.; Hassert, R.; Pagel, M.; Risselada, H. J.; Naumov, S.; Beck-Sickinger, A. G.; Abel, B. Peptides@mica: From Affinity to Adhesion Mechanism. Phys. Chem. Chem. Phys. 2016, 18 (34), 23516-23527.

(74) John, T.; Greene, G. W.; Patil, N. A.; Dealey, T. J. A.; Hossain, M. A.; Abel, B.; Martin, L. L. Adsorption of Amyloidogenic Peptides to Functionalized Surfaces Is Biased by Charge and Hydrophilicity. Langmuir 2019, 35 (45), 14522-14531.

(75) Wicklein, B.; Salazar-Alvarez, G. Functional Hybrids Based on Biogenic Nanofibrils and Inorganic Nanomaterials. J. Mater. Chem. A 2013, 1 (18), 5469-5478. 